This is a pre-copyedited, author-produced PDF of an article accepted for publication in Health Promotion International following peer review. The version of record Sharp P, Stolp S, Bottorff JL, Oliffe JL, Hunt K \& Caperchione CM (2020) Can lifestyle interventions improve Canadian men's mental health? Outcomes from the HAT TRICK programme. Health Promotion International, Volume 36, Issue 4, August 2021, Pages 943-951 is available online at: https://doi.org/10.1093/heapro/daaa120

\title{
Can lifestyle interventions improve Canadian men's mental health? Outcomes from the HAT TRICK program
}

Paul Sharp ${ }^{1 *}$, Sean Stolp ${ }^{1}$, Joan L. Bottorff ${ }^{2,3}$, John L. Oliffe ${ }^{4,5}$, Kate Hunt ${ }^{6}$, Cristina M.

Caperchione $^{1}$

\section{Author Affiliations:}

${ }^{1}$ School of Sport, Exercise and Rehabilitation, University of Technology Sydney, Sydney, NSW, Australia.

${ }^{2}$ Institute for Healthy Living and Chronic Disease Prevention, University of British

Columbia, Kelowna, BC, Canada

${ }^{3}$ School of Nursing, University of British Columbia, Kelowna, BC, Canada

${ }^{4}$ School of Nursing, University of British Columbia, Vancouver, BC, Canada

${ }^{5}$ Department of Nursing, University of Melbourne, Melbourne, Victoria, Australia

${ }^{6}$ Institute for Social Marketing and Health, University of Stirling, Stirling, UK

\section{*Corresponding Author:}

Paul Sharp

School of Sport, Exercise and Rehabilitation

Faculty of Health

University of Technology Sydney

Driver Avenue, Moore Park NSW 2021

PO Box 123. Broadway NSW 2007

email: paul.sharp@uts.edu.au

phone: +61 (02) 95145208

Keywords: Men; lifestyle intervention; depression risk, quality of life; physical activity

Word count: 5,560 (4060 words inc. references +3 tables) 
Registration: This trial is registered with clinicaltrials.gov [NCT03059199] and the International Clinical Trials Registry Platform [ISRCTN43361357].

Funding Sources: This work is supported by the Canadian Cancer Society [grant number 704230]; the Canadian Men's Health Foundation; a Tier 1 Canada Research Chair in Men's Health Promotion [to J.L.O.]; and the Australian Government Research Training Program Scholarship [to P.S.].

Conflict of Interest: All authors declare no conflict of interest.

Human Rights: Ethical approval for this trial was obtained from the University of British Columbia Okanagan Behavioral Research Ethics Board [\#H1600736].

Informed Consent: Informed consent was obtained from all individual participants included in the study. 


\section{ABSTRACT}

Engaging men in mental health promotion can be difficult because of reticence about helpseeking, especially for gender neutral programs. Developments in men's health research has pointed to the success of gender-sensitised programs to increase men's engagement in healthy lifestyle interventions targeting physical activity and healthy eating; however, less is known about the impact of these interventions on men's mental health. This study explored changes to men's depression risk and health-related quality of life at post-intervention (12 weeks) and 9-month follow-up, after participating in HAT TRICK, a gender-sensitised lifestyle intervention for overweight men. Participants completed validated self-report measures of mental health at baseline, post-intervention (12 weeks), and 9-month follow-up. Men's scores on the Male Depression Risk Scale (MDRS) and the SF-12 questionnaire, including physical health (PH12) and mental health (MH12) composite scores, were analysed using mixed linear models to assess linear trends. At baseline, men $(\mathrm{N}=62)$ had a mean age of $50.98(\mathrm{SD}=$ 10.09) years and BMI of $35.87(\mathrm{SD}=5.51) \mathrm{kg} / \mathrm{m}^{2}$. Results show that both the MDRS and the MH12 showed improvements in participants' mental health, with significant linear trends ( $p$ $=.003 ; p=.003)$ qualified with significant quadratic trends over time $(p=.02 ; p=.03)$. There were no significant changes in the PH12 over time. Gender-sensitised programs for overweight men, such as HAT TRICK, are a promising approach to positively influence components of men's mental health, with the potential for sustained improvements over the long-term. 


\section{INTRODUCTION}

Poor mental health among men, including depression and anxiety, is a leading contributor to men's burden of disease globally (Baker et al., 2014). It is reported that almost $10 \%$ of Canadian men will experience significant mental health challenges during their life, with approximately one million Canadian men suffering from major depression each year (Statistics Canada, 2018, Gravel et al., 2005). Confounding men's poor health outcomes is the bidirectional relationship between excess body weight and poor mental health outcomes (Pan et al., 2012). For instance, overweight and obesity is associated with decreased mental health and well-being and recursively, stress, anxiety, and depression may lead to unhealthy behaviours (e.g., poor diet, physical inactivity, social isolation) and weight gain (Errisuriz et al., 2016, Mouchacca et al., 2013).

Men's mental health promotion is challenged by gender-related barriers. For example, traditional masculine values of stoicism and independence can contribute to men's reticence towards help-seeking behaviours and formal psychological support (Seidler et al., 2016, Wang et al., 2013). As a result, men may avoid taking action altogether or engage in alternative strategies including substance use to manage their mental health. Proudfoot et al. (Proudfoot et al., 2015) found that two of the most commonly reported strategies used by men for preventing and managing mental health symptoms were physical activity and healthy eating. There is convincing evidence that engaging in regular physical activity and healthy eating may help to prevent and manage stress, anxiety, and depression, and increase selfesteem and overall health-related quality of life (Balchin et al., 2016, Cooney et al., 2013, Jayakody et al., 2014, Stonerock et al., 2015, Vuillemin et al., 2005, Wijndaele et al., 2007). Thus, engaging men in healthy lifestyle change may provide unique opportunities to improve men's mental health while avoiding gender-related barriers for seeking help. 
Interest in men's health has led to the development of health promotion strategies targeted at engaging men in healthy lifestyle programs. Utilising a gender-sensitised approach, with consideration to men's values, preferences, and interests, has been found to support men with making health behaviour changes (Bottorff et al., 2015, Oliffe et al., 2019, Sharp et al., 2020b). For instance, gender-sensitised engagement strategies such as using a male-only group-based format and encouraging humour, banter, and friendly competition during these sessions have been identified as important elements to program uptake and sustainability (Bottorff, et al., 2015, Hunt et al., 2020, Hunt et al., 2013). There is also the potential for programs to be gender-transformative whereby improving health and changing negative gender norms at the same time (Greaves et al., 2014). The importance of the gendered-social space offered within these programs, providing a sense of social connection, belonging, and camaraderie, has proven to be crucial to men's engagement (Oliffe, et al., 2019). To date, results from intervention studies have focused on physical health and behavioural outcomes, demonstrating improvements in weight-related outcomes, physical activity, and diet (Gray et al., 2013, Petrella et al., 2017, Wyke et al., 2015). However, little is known about how such programs impact men's mental health. A recent systematic review examining the impact of lifestyle interventions on men's mental health found only nine studies for inclusion in the review (Drew et al., 2020). In addition to a limited number of eligible studies, only $26 \%$ reported significant mental health outcomes and the authors noted a lack of longitudinal follow-up for mental health outcomes.

Building on the success of gender-sensitised lifestyle interventions, the HAT TRICK program was developed to engage overweight, inactive men in physical activity, healthy eating and social connectedness. The program was delivered in collaboration with a major junior ice hockey team in the Canadian Hockey League. Primary outcomes, including changes to men's physical activity, diet, and social connectedness, from participating in HAT 
TRICK are reported elsewhere (Caperchione et al., 2020). Here, we explore secondary outcomes related to changes in mental health, including risk of depression and health-related quality of life among HAT TRICK participants.

\section{METHODS}

\section{Study Design}

This exploratory study utilised a pre-post, quasi-experimental design. The overarching aim of this pragmatic trial was to assess feasibility and explore an estimate of effectiveness; thus, in alignment with common practice for feasibility and pilot studies, a power calculation was not conducted (Billingham et al., 2013, Bell et al., 2018). A recruitment target of 60 participants (20 participants $\times$ three consecutive intervention deliveries) was chosen and three cohorts of men completed HAT TRICK between December 2016 and January 2018. Recruitment methods included two media releases (i.e., newsprint and radio), a projectspecific website, social media (e.g., Facebook posts shared on local pages, community event forums), advertising during ice hockey games and word of mouth. Interested individuals were encouraged to contact a member of the research team by email or telephone to confirm eligibility and enrol. Measures were taken on all men at baseline, post-intervention (12 week) and 9-month follow-up. Further details on HAT TRICK's recruitment and methodological protocol are detailed elsewhere (Caperchione et al., 2017, Sharp et al., 2020a). All participants provided informed consent prior to baseline assessments. Ethical approval was obtained from the University of British Columbia Okanagan Behavioral Research Ethics Committee (\#H1600736) and this trial is registered with clinicaltrials.gov (NCT03059199) and the International Clinical Trials Registry Platform (ISRCTN43361357).

\section{Sample}

To be eligible, men had to be over the age of 35 years; self-report less than 150 minutes of physical activity per week and have a body mass index (BMI) of over $25 \mathrm{~kg} / \mathrm{m}^{2}$ 
with a pant waist size of 38 " or greater. All participants were screened using the Physical Activity Readiness Questionnaire (PAR-Q+) and required to seek medical clearance from a physician if any underlying concerns were identified (Canadian Society for Exercise Physiology, 2012).

\section{HAT TRICK Intervention}

HAT TRICK is a 12-week intervention focused on three specific components including physical activity, diet, and social connectedness. Content was gender-sensitised, drawing on constructs of Social Cognitive Theory (Bandura, 1986) and Self-Determination Theory (Teixeira et al., 2012), and incorporated gender-based approaches such as humour, positive banter, and strength-based messaging (Bottorff, et al., 2015). Throughout the program there was an emphasis on promoting social connectedness by fostering a sense of teamwork and camaraderie among the men during group-based activities and friendly competition. Small group activities were designed to encourage participants to share their experiences and challenges with making healthy changes as well as provide feedback and support to their peers. Components of the HAT TRICK intervention have been detailed elsewhere (Caperchione, et al., 2017) and are summarised here:

- Weekly 90-minute, group-based sessions including targeted health education regarding physical activity, healthy eating, and behaviour change techniques (i.e., goal setting, self-monitoring).

- Progressive physical activity (i.e., increasing in duration and intensity on a weekly basis) inclusive of a variety of 'men-friendly' activities (e.g., walking, resistance training, ball hockey).

- Weekly challenges, including physical activity (e.g., increase weekly steps by 500/day) and healthy eating goals (e.g., choose water instead of sugary drinks on at least 3 days of the week). 
- HAT TRICK Playbook, a print-based informational resource booklet with tailored messaging concerning physical activity, healthy eating and behaviour change as techniques (e.g., goal setting, social support, self-monitoring) well as weekly physical activity and dietary tracking logs.

- FitBit Charge HRTM, used to self-monitor daily steps, heart rate, and minutes of moderate-to-vigorous physical activity.

\section{Measures}

\section{Participant characteristics}

Age, ethnic background, level of education, marital status, main activity (i.e., full time or part time work, caring for household, retired), occupation and household income were selfreported. Weight and height were measured at baseline by a trained research assistant and used to calculate BMI to assess eligibility requirements.

\section{Depression Risk}

Participant mental health was assessed using the Male Depression Risk Scale (MDRS) (Rice et al., 2013). The MDRS is a valid and reliable 22-item, 7 point Likert scale questionnaire (0 (not at all) -7 (almost always)). The total score of the MDRS is a summation of six subscales: emotional suppression, drug use, alcohol use, anger and aggression, somatic symptoms and risk-taking. Higher scores indicate a greater risk of depression.

\section{Health-related Quality of Life}

Quality of life was assessed using the SF-12 (Ware Jr et al., 1996), which contains two overarching components (physical health and mental health), and has been shown to be valid and reliable in a number of populations (Burdine et al., 2000). The mental health component (MH12) is comprised of four components: vitality, social functioning, roleemotional and mental health. The physical health (PH12) component is also comprised of 
four components: physical functioning role-physical, bodily pain and general health. Components are scored from 0 to 100, with higher scores indicating better health.

\section{Data Analysis}

Demographic data were analysed descriptively. Dependent variable linear and quadratic trends were analysed across baseline, post-program and 9-month follow-up using multi-level modelling (MLM) (Casals et al., 2014). Linear terms were used to assess change over time on the dependent variable and, to qualify these trends, quadratic terms were used to assess if the change accelerated or decelerated over time. Descriptive statistics were used to identify variables with outliers ( \pm 3 standard deviations from the mean). Initial modelling began with linear and quadratic components and a random effect for the intercept estimated on the linear term, to assess the shape of growth trajectories and to assess whether the means of the MDRS, MH12 and PH12 measures differed across time. Restricted maximum likelihood was used for the estimation method. Covariance estimates were assessed to determine if a random participant intercept was appropriate for the model. Models were rerun if covariance estimates suggested no between participant variability. Differing covariance structures were assessed in an attempt to define the best model as by the smallest Akiaike's Information Criterion (AIC). Analyses were run using SPSS version 26.

\section{RESULTS}

\section{Descriptive Characteristics}

Sixty-two male participants with a mean age of $50.98(\mathrm{SD}=10.09)$ years and BMI of $35.87(\mathrm{SD}=5.51) \mathrm{kg} / \mathrm{m}^{2}$ completed measures at baseline; 58 completed post program measures, of which 54 completed 9-month follow-up measures. Full descriptive characteristics are shown in Table 1. Means and standard deviations for dependent variables are shown seen in Table 2. 
Table 1. Descriptive Characteristics of Men at Baseline $(n=62)$

\begin{tabular}{|c|c|c|c|}
\hline \multicolumn{2}{|l|}{ Measure } & $\begin{array}{l}\text { Frequency } \\
\text { (n) }\end{array}$ & \multirow{2}{*}{$\begin{array}{l}\text { Percentage } \\
(\%) \\
1.6\end{array}$} \\
\hline Highest Level of & Some high school or less & 1 & \\
\hline \multirow[t]{2}{*}{ Education } & High school diploma & 13 & 20.9 \\
\hline & College or technical / University degree & 48 & 77.4 \\
\hline Household Income & $\$ 25,000-\$ 49,999$ & 6 & 9.7 \\
\hline \multirow{2}{*}{ Before Taxes } & $\$ 50,000-\$ 99,999$ & 26 & 41.9 \\
\hline & $\$ 100,000$ or more & 30 & 48.4 \\
\hline \multirow[t]{4}{*}{ Main Activity } & Full time work & 50 & 80.6 \\
\hline & Part time work & 1 & 1.6 \\
\hline & Caring for family/managing & 11 & 17.7 \\
\hline & household/retired & & \\
\hline \multirow[t]{3}{*}{ Marital Status } & Married/domestic partnership & 54 & 87.1 \\
\hline & Divorced/separated & 5 & 8.1 \\
\hline & Single/never married/widowed & 3 & 4.8 \\
\hline \multirow[t]{5}{*}{ Ethnic Background } & Caucasian & 57 & 91.9 \\
\hline & Metis & 1 & 1.6 \\
\hline & South Asian & 1 & 1.6 \\
\hline & West Asian & 1 & 1.6 \\
\hline & Other & 2 & 3.2 \\
\hline \multirow[t]{4}{*}{ MDRS } & Low Risk & 49 & 79.0 \\
\hline & Elevated Risk & 12 & 19.4 \\
\hline & High Risk & 1 & 1.6 \\
\hline & Extreme Risk & 0 & 0.0 \\
\hline \multirow[t]{4}{*}{ BMI Category } & Overweight & 5 & 8.1 \\
\hline & Obese Class 1 & 21 & 33.9 \\
\hline & Obese Class 2 & 25 & 40.3 \\
\hline & Obese Class 3 & 11 & 17.7 \\
\hline
\end{tabular}


Table 2. Means, Standard Deviations and Effect Sizes of Dependent Variables

\begin{tabular}{|c|c|c|c|c|}
\hline \multirow[t]{3}{*}{ Measure } & Baseline & 12-weeks & 9-month Follow-up & Cohen's d \\
\hline & Mean (SD) & Mean (SD) & Mean (SD) & Baseline vs (12 \\
\hline & $(n=62)$ & $(n=57)$ & $(n=54)$ & weeks -9 month) \\
\hline $\mathrm{MDRS}^{\mathrm{L}, \mathrm{Q}}$ & $21.48(13.6)$ & $16.67(13.12)$ & $17.31(13.64)$ & $(0.35-0.30)$ \\
\hline PH12 & $48.31(6.73)$ & $49.12(7.04)$ & $49.98(6.21)$ & $(0.11-0.26)$ \\
\hline $\mathrm{MH} 12^{\mathrm{L}, \mathrm{Q}}$ & $52.56(6.00)$ & $54.34(5.65)$ & $54.6(5.00)$ & $(0.31-0.29)$ \\
\hline
\end{tabular}

\section{Depression Risk}

After the removal of outliers, initial multilevel modelling showed total MDRS had significant linear decreasing trend over time $(p=.003)$, qualified with significant quadratic trend $(p=.02)$. See Table 3 .

\section{Health Related Quality of Life}

The MH12 showed significant linear growth over time $(p=.003)$, qualified with significant quadratic trend $(p=.03)$. The PH12 showed no significant linear growth over time. See Table 3. 
Table 3. Linear and Quadratic Trends of Dependent Variables

\begin{tabular}{llccc}
\hline \multicolumn{2}{c}{ Dependent Variable } & $t(\mathrm{df})$ & \multicolumn{2}{c}{ Estimates of Fixed Effects } \\
\cline { 3 - 4 } & & & Estimate & $95 \%$ CI \\
& & & (Std. Error $)$ & \\
\hline MDRS & Intercept & $12.42(91.40)$ & $21.81(1.76)$ & $\mathbf{1 8 . 3 3}$ to $\mathbf{2 5 . 3 0}$ \\
& Linear & $-3.13(73.07)$ & $-7.37(2.35)$ & $\mathbf{- 1 2 . 2 0}$ to $\mathbf{- 2 . 6 8}$ \\
& Quadratic & $2.47(59.03)$ & $2.74(1.11)$ & $\mathbf{0 . 5 5}$ to 4.99 \\
\hline PH12 & Intercept & $57.42(124.71)$ & $48.31(0.84)$ & 46.65 to 49.98 \\
& Linear & $0.59(102.60)$ & $0.78(1.31)$ & -1.81 to 3.37 \\
& Quadratic & $0.05(93.99)$ & $0.03(0.61)$ & -1.17 to 1.23 \\
\hline MH12 & Intercept & $74.55(96.70)$ & $52.56(0.70)$ & $\mathbf{5 1 . 0 1}$ to 54.01 \\
& Linear & $3.06(105.56)$ & $2.58(0.84)$ & $\mathbf{0 . 8 4}$ to 4.59 \\
& Quadratic & $-2.14(98.48)$ & $-0.83(0.39)$ & $\mathbf{- 1 . 8 3}$ to $\mathbf{- 0 . 0 3}$ \\
\hline
\end{tabular}




\section{DISCUSSION}

HAT TRICK was designed as a gender-sensitised intervention aimed at attracting and engaging overweight, inactive men in healthy lifestyle change (i.e., physical activity, healthy eating, social connectedness). We show here that mental health improved after taking part in HAT TRICK, whereby participants' depression risk decreased and their mental health improved immediately post-intervention and improvements were maintained at 9-month follow-up. These findings contribute to emergent evidence that lifestyle interventions can help to improve mental health in men (Drew, et al., 2020, Kelly et al., 2019). Despite HAT TRICK not directly targeting mental health through education or intervention, these results are notable given the longstanding challenges for targeting men's mental health promotion. In particular, among a sample of overweight men, where mental health is challenged, efforts targeted at improving and maintaining mental health are highly relevant. In light of these findings, we provide suggestions and considerations for future work in this important and developing area.

Men's mental illness endures significant societal and self-stigma, and by extension programs naming an explicit focus on mental health promotion can be off putting for men (Oliffe et al., 2016). A benefit of HAT TRICK and similar men-only, lifestyle group-based programs is that the emphasis on physical activity was familiar and congruent with many men's masculine values. Moreover, leading with the 'doing' of physical activity appealed to men and resonated with their experiences of team-based endeavours. These familiar 'masculine' milieus afforded the space and comradery to bolster mental health and reduce depression risk. We suggest that not forefronting mental health as a program goal was important to HAT TRICK's recruitment efforts; rather, building group rapport within the program may (eventually) afford opportunities to engage men in content and conversations about men's stresses and their strategies for addressing such challenges. In line with Oliffe et 
al. (Oliffe, et al., 2019), the language of HAT TRICK should work with participants' own literacy ('stress') rather than invoking medical constructs (mental health and illness). Of course, the timing for addressing men's mental health is important, and based on our process evaluation data (Sharp et al., 2018) men's openness to discussing such matters was more evident in the latter half of the program (weeks 6-12). These findings respond, in part, to Drew et al.'s (Drew, et al., 2020) call for research to better understand connections between lifestyle interventions and men's mental health and align with others who have begun to include mental health components (e.g., stress management workshops) within the later portion of intervention delivery (Carroll et al., 2018). Future work might usefully explore the utility of these mental health literacy and timing insights as well as the acceptability of other program components, including sportsmen testimonials and vignettes (e.g., well-known hockey figures sharing personal experiences concerning their own mental health and wellbeing) to engage men with avenues for reaching out to other men who might be experiencing mental health challenges.

While mean scores for mental health indicators were not at elevated risk at baseline, observed improvements in men's mental health over the course of this community-based program and were maintained at 9 month follow-up are noteworthy. Importantly, these results were obtained with a group of overweight men who are known to be at high risk for mental health issues. In addition, these findings suggest that tailored lifestyle programs like HAT TRICK provide a promising avenue for promotion of men's mental wellbeing that may prevent or mitigate depression and other mental health issues in the future. It is difficult to speculate on the causal mechanisms underpinning these improvements in men's mental health. Despite robust evidence of the positive effects of lifestyle interventions focusing on physical activity and diet on psychological health in both healthy populations and those experiencing mental health problems (Dale et al., 2014), the causal mechanisms have not 
been clearly established. Nevertheless, there are several possible factors that may explain improvements in men's mental health observed in this study. While physical activity is associated with several physiological mechanisms (e.g., neurotransmitter release, inflammation reduction) that may affect mental health (Kandola et al., 2019, Teychenne et $a l ., 2020)$, it is also clear that other aspects of the physical activity experience may influence mental health outcomes (e.g., sense of belonging, social interactions)(Biddle et al., 2007). The therapeutic value of peer support enabled through this gender-sensitised intervention has been observed in other community-based men's health promotion programs (Oliffe, et al., 2019). It is also possible that increases in self-efficacy associated with simultaneous improvements in physical activity and diet may have extended to other life changes as well as the ability to use physical activity as a coping mechanism post intervention. As well, the use of a variety of behaviour change techniques (e.g., action planning, goal setting, selfmonitoring) to promote maintenance of lifestyle changes may have contributed longer-term mental health benefits. Since HAT TRICK offers an important alternative that is acceptable to men who may be at risk for mental health issues, there is value in identifying essential intervention components associated with observed improvements in mental health in future research.

The study has a number of strengths. First, using validated instruments to measure mental health and with follow-up to 9 months, we have been able to show substantial potential mental health gains through participation in a 12-week group-based program designed to promote healthy lifestyle changes (including increased physical activity) and social connectedness with others (in particular men with a shared interest in ice hockey). Secondly, attrition between data collection points was low; $94 \%$ and $87 \%$ of those measure at baseline took part in the post-program and 9-month follow-up measurement. It is plausible 
that the small minority who did not provide follow-up data did not experience improvements in their mental health.

The study also has limitations inherent in its pre/post study design. Here we report findings from an exploratory study of men attending the first deliveries of the program at one club. To fully test whether the HAT TRICK program has sustained benefits for the mental health of men who are overweight and inactive, a multi-site randomised controlled trial powered to detect a difference in a specific measure of mental health (as the primary outcome) would be required. This study suggests that there would be merit in undertaking such a trial. Further, participants in this study were predominately Caucasian, potentially limiting transferability. Thoughtful consideration should be given to recruiting a more diverse sample whose access to such programs may be inhibited by health inequities.

\section{CONCLUSION}

It is widely acknowledged that it is difficult to engage men in many health-promoting programs, although there have been recent notable successes for programs which take a gender-sensitised approach to recruitment, and in content and style of delivery. Evidence suggests that men's reluctance to engage with treatment or health-promoting services is likely to be most pronounced for mental health issues. This program, with its 'male-friendly', action-focused approach, which fosters social connections, positive banter and shared enjoyment of program components and context, appears to have great potential. Without an explicit focus on mental health, it shows improvements which could have significant benefit at the individual and population level, whilst simultaneously shifting a culture of silence amongst men about mental health issues. Future research could explore the most crucial pathways in a logic model to better understand which components of the program' content 
and delivery drive these positive changes, and whether there are further components which could or should be added to maximise mental health gains. 


\section{REFERENCES}

Baker, P., Dworkin, S. L., Tong, S., Banks, I., Shand, T. and Yamey, G. (2014) The men's health gap: Men must be included in the global health equity agenda. Bulletin of the World Health Organization, 92, 618-620.

Balchin, R., Linde, J., Blackhurst, D., Rauch, H. L. and Schönbächler, G. (2016) Sweating away depression? The impact of intensive exercise on depression. Journal of affective disorders, 200, 218-221.

Bandura, A. (1986) Social foundations of thought and action. Englewood Cliffs, NJ, 1986.

Bell, M. L., Whitehead, A. L. and Julious, S. A. (2018) Guidance for using pilot studies to inform the design of intervention trials with continuous outcomes. Clinical epidemiology, 10, 153.

Biddle, S. J. and Mutrie, N. (2007) Psychology of physical activity: Determinants, well-being and interventions. Routledge.

Billingham, S. A., Whitehead, A. L. and Julious, S. A. (2013) An audit of sample sizes for pilot and feasibility trials being undertaken in the united kingdom registered in the united kingdom clinical research network database. BMC medical research methodology, 13, 104.

Bottorff, J. L., Seaton, C. L., Johnson, S. T., Caperchione, C. M., Oliffe, J. L., More, K., Jaffer-Hirji, H. and Tillotson, S. M. (2015) An updated review of interventions that include promotion of physical activity for adult men. Sports Medicine, 45, 775-800. 10.1007/s40279014-0286-3.

Burdine, J. N., Felix, M. R., Abel, A. L., Wiltraut, C. and Musselman, Y. (2000) The sf-12 as a population health measure: An exploratory examination of potential for application. Health services research, $35,885$.

Canadian Society for Exercise Physiology (2012) Physial activity and readiness questionnaire for everyone: Par-q+.

http://www.csep.ca/cmfiles/publications/parq/parqplussept2011version_all.pdf.

Caperchione, C. M., Bottorff, J. L., Oliffe, J. L., Johnson, S. A., Hunt, K., Sharp, P., Fitzpatrick, K. M., Price, R. and Goldenberg, S. L. (2017) The hat trick program for improving physical activity, healthy eating and connectedness among overweight, inactive men: Study protocol of a pragmatic feasibility trial. BMJ Open.

Caperchione, C. M., Bottorff, J. L., Stolp, S., Sharp, P., Johnson, S. T., Oliffe, J. L. and Hunt, K. (2020) Positive lifestyle behavior changes among canadian men: Findings from the hat trick program. American Journal of Health Promotion, 0890117120957176.

Carroll, P., Harrison, M., Richardson, N., Robertson, S., Keohane, A., Kelly, L. and Donohoe, A. (2018) Evaluation of a gender-sensitive physical activity programme for inactive men in ireland: Protocol paper for a pragmatic controlled trial. Journal of Physical Activity Research, 3, 20-27.

Casals, M., Girabent-Farres, M. and Carrasco, J. L. (2014) Methodological quality and reporting of generalized linear mixed models in clinical medicine (2000-2012): A systematic review. PloS one, 9.

Cooney, G. M., Dwan, K., Greig, C. A., Lawlor, D. A., Rimer, J., Waugh, F. R., McMurdo, M. and Mead, G. E. (2013) Exercise for depression. Cochrane database of systematic reviews. 
Dale, H., Brassington, L. and King, K. (2014) The impact of healthy lifestyle interventions on mental health and wellbeing: A systematic review. Mental Health Review Journal.

Drew, R. J., Morgan, P. J., Pollock, E. R. and Young, M. D. (2020) Impact of male-only lifestyle interventions on men's mental health: A systematic review and meta-analysis. Obesity Reviews.

Errisuriz, V. L., Pasch, K. E. and Perry, C. L. (2016) Perceived stress and dietary choices: The moderating role of stress management. Eating Behaviors, 22, 211-216.

Gravel, R. and Béland, Y. (2005) The canadian community health survey: Mental health and well-being. The Canadian Journal of Psychiatry, 50, 573-579.

Gray, C. M., Hunt, K., Mutrie, N., Anderson, A. S., Leishman, J., Dalgarno, L. and Wyke, S. (2013) Football fans in training: The development and optimization of an intervention delivered through professional sports clubs to help men lose weight, become more active and adopt healthier eating habits. BMC Public Health, 13, 232. 10.1186/1471-2458-13-232. Greaves, L., Pederson, A. and Poole, N. (2014) Making it better: Gender transformative health promotion. Canadian Scholars' Press.

Hunt, K., McCann, C., Gray, C. M., Mutrie, N. and Wyke, S. (2013) "You've got to walk before you run": Positive evaluations of a walking program as part of a gender-sensitized, weight-management program delivered to men through professional football clubs. Health psychology, 32, 57-65. 10.1037/a0029537.

Hunt, K., Wyke, S., Bunn, C., Donnachie, C., Reid, N. and Gray, C. M. (2020) Scale-up and scale-out of a gender-sensitized weight management and healthy living program delivered to overweight men via professional sports clubs: The wider implementation of football fans in training (ffit). International Journal of Environmental Research and Public Health, 17, 584.

Jayakody, K., Gunadasa, S. and Hosker, C. (2014) Exercise for anxiety disorders: Systematic review. British Journal of Sports Medicine, 48, 187-196.

Kandola, A., Ashdown-Franks, G., Hendrikse, J., Sabiston, C. M. and Stubbs, B. (2019)

Physical activity and depression: Towards understanding the antidepressant mechanisms of physical activity. Neuroscience \& Biobehavioral Reviews, 107, 525-539.

Kelly, L., Harrison, M., Richardson, N., Carroll, P., Robertson, S., Keohane, A. and Donohoe, A. (2019) The impact of a gender-specific physical activity intervention on the fitness and fatness profile of men in ireland. European Journal of Public Health, 29, 11541160 .

Mouchacca, J., Abbott, G. R. and Ball, K. (2013) Associations between psychological stress, eating, physical activity, sedentary behaviours and body weight among women: A

longitudinal study. BMC public health, 13, 828.

Oliffe, J. L., Ogrodniczuk, J. S., Gordon, S. J., Creighton, G., Kelly, M. T., Black, N. and Mackenzie, C. (2016) Stigma in male depression and suicide: A canadian sex comparison study. Community mental health journal, 52, 302-310.

Oliffe, J. L., Rossnagel, E., Bottorff, J. L., Chambers, S. K., Caperchione, C. and Rice, S. M. (2019) Community-based men's health promotion programs: Eight lessons learnt and their caveats. Health promotion international.

Pan, A., Keum, N., Okereke, O. I., Sun, Q., Kivimaki, M., Rubin, R. R. and Hu, F. B. (2012) Bidirectional association between depression and metabolic syndrome: A systematic review and meta-analysis of epidemiological studies. Diabetes care, 35, 1171-1180.

Petrella, R. J., Gill, D. P., Zou, G., De Cruz, A., Riggin, B., Bartol, C., Danylchuk, K., Hunt, K., Wyke, S., Gray, C. M. and Bunn, C. (2017) Hockey fans in training: A pilot pragmatic randomized controlled trial. Journal of American College of Sports Medicine.

Proudfoot, J., Fogarty, A. S., McTigue, I., Nathan, S., Whittle, E. L., Christensen, H., Player, M. J., Hadzi-Pavlovic, D. and Wilhelm, K. (2015) Positive strategies men regularly use to 
prevent and manage depression: A national survey of australian men. BMC Public Health, 15, 1135.

Rice, S. M., Fallon, B. J., Aucote, H. M. and Möller-Leimkühler, A. M. (2013) Development and preliminary validation of the male depression risk scale: Furthering the assessment of depression in men. Journal of affective disorders, 151, 950-958.

Seidler, Z. E., Dawes, A. J., Rice, S. M., Oliffe, J. L. and Dhillon, H. M. (2016) The role of masculinity in men's help-seeking for depression: A systematic review. Clinical Psychology Review, 49, 106-118.

Sharp, P., Bottorff, J. L., Hunt, K., Oliffe, J. L., Johnson, S. T., Dudley, L. and Caperchione, C. M. (2018) Men's perspectives of a gender-sensitized health promotion program targeting healthy eating, active living, and social connectedness. American Journal of Men's Health, 0 , 1557988318799159. 10.1177/1557988318799159.

Sharp, P., Bottorff, J. L., Oliffe, J. L., Hunt, K. and Caperchione, C. M. (2020a) Process evaluation of hat trick: Feasibility, acceptability, and opportunities for program refinement Health Education Research. 10.1093/her/cyaa029.

Sharp, P., Spence, J. C., Bottorff, J. L., Oliffe, J. L., Hunt, K., Vis-Dunbar, M. and Caperchione, C. M. (2020b) One small step for man, one giant leap for men's health: A metaanalysis of behaviour change interventions to increase men's physical activity. British Journal of Sports Medicine.

Statistics Canada (2018) Canadian community health survey. https://www23.statcan.gc.ca/imdb/p2SV.pl?Function=getSurvey\&SDDS=3226.

Stonerock, G. L., Hoffman, B. M., Smith, P. J. and Blumenthal, J. A. (2015) Exercise as treatment for anxiety: Systematic review and analysis. Annals of Behavioral Medicine, 49, 542-556.

Teixeira, P. J., Carraça, E. V., Markland, D., Silva, M. N. and Ryan, R. M. (2012) Exercise, physical activity, and self-determination theory: A systematic review. International Journal of Behavioral Nutrition and Physical Activity, 9, 78.

Teychenne, M., White, R. L., Richards, J., Schuch, F. B., Rosenbaum, S. and Bennie, J. A. (2020) Do we need physical activity guidelines for mental health: What does the evidence tell us? Mental Health and Physical Activity, 18, 100315.

Vuillemin, A., Boini, S., Bertrais, S., Tessier, S., Oppert, J.-M., Hercberg, S., Guillemin, F. and Briançon, S. (2005) Leisure time physical activity and health-related quality of life. Preventive medicine, 41, 562-569.

Wang, Y., Hunt, K., Nazareth, I., Freemantle, N. and Petersen, I. (2013) Do men consult less than women? An analysis of routinely collected uk general practice data. BMJ Open, 3. Ware Jr, J. E., Kosinski, M. and Keller, S. D. (1996) A 12-item short-form health survey: Construction of scales and preliminary tests of reliability and validity. Medical care, 220233.

Wijndaele, K., Matton, L., Duvigneaud, N., Lefevre, J., De Bourdeaudhuij, I., Duquet, W., Thomis, M. and Philippaerts, R. M. (2007) Association between leisure time physical activity and stress, social support and coping: A cluster-analytical approach. Psychology of Sport and Exercise, 8, 425-440.

Wyke, S., Hunt, K., Gray, C. M., Fenwick, E., Bunn, C., Donnan, P. T., Rauchhaus, P., Mutrie, N., Anderson, A. S., Boyer, N., Brady, A., Grieve, E., White, A., Ferrell, C., Hindle, E. and Treweek, S. (2015) Football fans in training (ffit): A randomised controlled trial of a gender-sensitised weight loss and healthy living programme for men-end of study report. Public Health Research, 3, 1-129. NBK273998. 